valves were involved in 9 and 8 cases, respectively. Cardiac involvement was silent in $36.4 \%$ and occurred as an initial presenting symptom of SLE in $9.1 \%$ cases. Biologically, patients with cardiac involvement had higher levels of CRP and anti dsDNA, and lower levels of complement C3 compared to patients with no cardiac involvement; while SLE activity was the only significant predictor for cardiac involvement $($ Beta $=0.654 ; \mathrm{p}=0.020)$

Conclusions Cardiac complications are common (1 out of 3 times). They are predicted by high SLE activity and antidsDNA, CRP and low C3 levels. Reguiar echocardiography is erommended for Patients with high SLE activity.

\section{OUTCOMES OF 847 CHILDHOOD-ONSET SYSTEMIC LUPUS ERYTHEMATOUSUS PATIENTS IN THREE AGE GROUPS}

${ }^{1} \mathrm{~L}$ Campos*, ${ }^{2} \mathrm{~S}$ Lopes, ${ }^{2} \mathrm{~N}$ Gormezano, ${ }^{3} \mathrm{R}$ Gomes, ${ }^{2} \mathrm{~N}$ Aikawa, ${ }^{2} \mathrm{R}$ Pereira, ${ }^{4} \mathrm{M}$ Terreri, ${ }^{5} \mathrm{C}$ Magalhaes, ${ }^{6} \mathrm{E}$ Okuda, ${ }^{4} \mathrm{~A}$ Sakamoto, ${ }^{3} \mathrm{~A}$ Sallum, ${ }^{7} \mathrm{~S}$ Appenzeller, ${ }^{8} \mathrm{~V}$ Ferriani, ${ }^{9} \mathrm{C}$ Barbosa, ${ }^{10} \mathrm{~S}$ Lotufo, ${ }^{4} \mathrm{~L}$ Andrade, ${ }^{2} \mathrm{E}$ Bonfa, ${ }^{3} \mathrm{C}$ Silva. ${ }^{1}$ SAO PAULO, Brazil, ${ }^{2}$ Faculdade de Medicina da Universidade de São Paulo, Division of Rheumatology, Sao Paulo, Brazil; ${ }^{3}$ Children's Institute- Faculdade de Medicina da Universidade de São Paulo, Paediatric Rheumatology Unit, Sao Paulo, Brazil; ${ }^{4}$ Universidade Federal de São Paulo, Paediatric Rheumatology Unit, Sao Paulo, Brazil; ${ }^{5}$ São Paulo State University UNESP - Faculdade de Medicina de Botucatu, Paediatric Rheumatology Division, Sao Paulo, Brazil; ${ }^{6}$ Irmandade da Santa Casa de Misericórdia de São Paulo, Paediatric Rheumatology Unit, Sao Paulo, Brazil ; ' State University of Campinas UNICAMP, Paediatric Rheumatology Unit, Sao Paulo, Brazil; ${ }^{8}$ Ribeirão Preto Medical School - University of São Paulo, Paediatric Rheumatology Unit, Sao Paulo, Brazil; ${ }^{9}$ Hospital Infantil Darcy Vargas, Paediatric Rheumatology Unit, Sao Paulo, Brazil; ${ }^{10}$ Hospital Menino Jesus, Paediatric Rheumatology Unit, Sao Paulo, Brazil

\subsection{6/lupus-2017-000215.128}

Background and aims To assess outcomes of childhood systemic lupus erythematosus (cSLE) in three different age groups evaluated at last visit: group A early-onset disease( $<6$ years), group $B$ school age $(\geq 6$ and $<12$ years $)$ and group $C$ adolescent ( $\geq 12$ and $<18$ years).

Methods Observational cohort study of 10 Paediatric Rheumatology centres, including 847 cSLE patients.

Results Group A had 39 (4\%), B 395 (47\%) and C 413 (49\%). Median disease duration was higher in group A compared to groups B and $\mathrm{C}[8.3(0.1-23.4)$ vs. $6.2(0-17)$ vs. 3.3 $(0-14.6)$ years, $\mathrm{p}<0.0001]$. The median SLICC/ACR-DI[0 (0$9)$ vs. $0(0-6)$ vs. $0(0-7), p=0.065]$ was comparable in all groups. Further analysis of organ/system damage revealed that frequencies of neuropsychiatric(21\% vs. $10 \%$ vs. $7 \%$, $\mathrm{p}=0.007)$, skin $(10 \%$ vs. $1 \%$ vs. $3 \%, \mathrm{p}=0.002)$ and peripheral vascular involvements(5\% vs. $3 \%$ vs. $0.3 \%, p=0.008)$ were more frequent in group A compared to B and C. Frequencies of severe cumulative lupus manifestations such as nephritis, thrombocytopenia and autoimmune hemolytic anaemia were similar in all groups $(\mathrm{p}>0.05)$. Mortality rate was higher in group A compared to groups B and C(15\% vs. $10 \%$ vs. $6 \%$, $\mathrm{p}=0.028)$. Out of 69 deaths, 33/69 (48\%) occurred within the first two years after diagnosis. Infections accounted for $54 / 69(78 \%)$ of the deaths and 38/54 (70\%) had concomitant disease activity.

Conclusions: This large multicenter study provided evidence that early-onset cSLE group had distinct outcomes, with higher mortality rate and neuropsychiatric/vascular/skin organ damages in spite of comparable frequencies of severe cumulative lupus manifestations. We also identified that overall death in cSLE patients was an early event mainly attributed to infection associated with disease activity.

\section{9 PANNICULITIS IN CHILDHOOD-ONSET SYSTEMIC LUPUS ERYTHEMATOSUS: A MULTICENTRIC COHORT STUDY}

'L Campos*, ${ }^{2} \mathrm{M}$ Verdier, ${ }^{2} \mathrm{P}$ Anuardo, ${ }^{2} \mathrm{~N}$ Gormezano, ${ }^{3} \mathrm{R}$ Romiti, ${ }^{4} \mathrm{~N}$ Aikawa, ${ }^{4} \mathrm{R}$ Pereira, ${ }^{5} \mathrm{M}$ Terreri, ${ }^{6} \mathrm{C}$ Magalhaes, ${ }^{2} \mathrm{~J}$ Ferreira, ${ }^{2} \mathrm{M}$ Silva, ${ }^{2} \mathrm{M}$ Ferriani, ${ }^{5} \mathrm{~A}$ Sakamoto, ${ }^{7} \mathrm{~V}$ Ferriani, ${ }^{8} \mathrm{M}$ Centeville, ${ }^{6} \mathrm{~J}$ Sato, ${ }^{9} \mathrm{M}$ Santos, ${ }^{4} \mathrm{E}$ Bonfa, ${ }^{2} \mathrm{C}$ Silva. ${ }^{1} \mathrm{SAO}$ PAULO, Brazil; ${ }^{2}$ Children's Institute- Faculdade de Medicina da Universidade de São Paulo, Paediatric Rheumatology Unit, Sao Paulo, Brazil; ${ }^{3}$ Faculdade de Medicina da Universidade de São Paulo, Division of Dermatology, Sao Paulo, Brazil; ${ }^{4}$ Faculdade de Medicina da Universidade de São Paulo, Division of Rheumatology, Sao Paulo, Brazil; ${ }^{5}$ Universidade Federal de São Paulo, Paediatric Rheumatology Unit, Sao Paulo, Brazil; ${ }^{6}$ São Paulo State University UNESP - Faculdade de Medicina de Botucatu, Paediatric Rheumatology Unit, Sao Paulo, Brazil; ${ }^{7}$ Ribeirão Preto Medical School - University of São Paulo, Paediatric Rheumatology Unit, Sao Paulo, Brazil; ${ }^{8}$ São Paulo State University of Campinas UNICAMP, Paediatric Rheumatology Unit, Sao Paulo, Brazil; ${ }^{9}$ Irmandade da Santa Casa de Misericórdia de São Paulo, Paediatric Department, Sao Paulo, Brazil

\subsection{6/lupus-2017-000215.129}

Background and Aims To evaluate prevalence, clinical manifestations, laboratory abnormalities, treatment and outcome in a multicenter cohort of childhood-onset systemic lupus erythematosus(cSLE) patients with and without panniculitis.

Methods Panniculitis was diagnosed due to painful subcutaneous nodules and/or plaques in deep dermis/subcutaneous tissues and lobular/mixed panniculitis with lymphocytic lobular inflammatory infiltrate in skin biopsy. Statistical analysis was performed using Bonferroni correction $(\mathrm{p}<0.004)$.

Results Panniculitis was observed in 6/847 (0.7\%) cSLE. Painful subcutaneous erythematosus and indurated nodules were observed in 6/6 panniculitis patients and painful subcutaneous plaques in $4 / 6$. Generalised distribution was evidenced in $3 / 6$ and localised in upper limbs in $2 / 6$ and face in 1/6. Histopathology features showed lobular panniculitis without vasculitis in $5 / 6$ (one of them had concomitant obliterative vasculopathy due to antiphospholipid syndrome) and panniculitis with vasculitis in $1 / 6$. Comparison between cSLE with panniculitis and 60 cSLE without panniculitis with same disease duration [2.75 $(0-11.4)$ vs. $2.83(0-11.8)$ years, $\mathrm{p}=0.297]$, showed higher frequencies of constitutional involvement (67\% vs. $10 \%$, $\mathrm{p}=0.003)$, leukopenia (67\% vs. $7 \%, \mathrm{p}=0.002)$ and median Creactive protein $(10.5$ vs. $0.5 \mathrm{mg} / \mathrm{L}, \mathrm{p}=0.001)$. Cutaneous atrophy and hyperpigmentation occurred in $83 \%$ of patients.

Conclusions Panniculitis is a rare skin manifestation of cSLE occurring in the first three years of disease with considerable sequelae. The majority of patients have concomitant mild lupus manifestations.

\section{NORMAL-PRESSURE HYDROCEPHALUS IN A NINE YEAR OLD FILIPINO FEMALE WITH SYSTEMIC LUPUS ERYTHEMATOSUS}

MT Collante*, C Bernal. University of Santo Tomas Hospital, Department of PediatricsSection of Paediatric Rheumatology, Manila, Philippines

\subsection{6/lupus-2017-000215.130}

Background and Aims Central nervous system involvement in paediatric systemic lupus erythematosus (SLE) is common, however, normal-pressure hydrocephalus is unusual.

Methods The medical records of a nine-year old Filipino female with SLE was reviewed.

Results Patient presented with prolonged fever, weight loss, generalised weakness, lapses in memory, and mood lability. She was poorly nourished, non-ambulatory, pale, febrile, and ill-looking. She was tachycardic, with normal blood pressure, 